
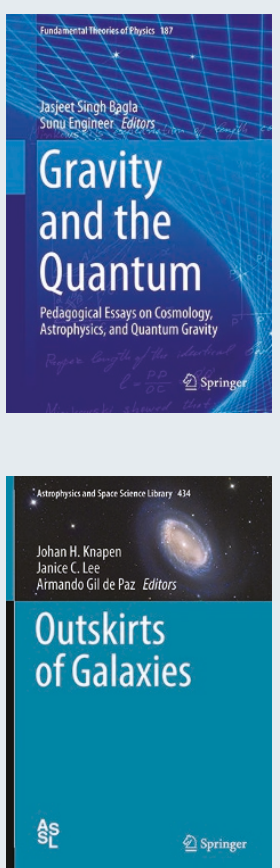

\section{Outskirts of Galaxies}

Edited by Johan H. Knapen, Janice C. Lee and Armando Gil de Paz SPRINGER NATURE: 2017. 362PP. £112.00

When astronomers think of galaxies, typically they focus by necessity on their central parts where enough stars shine sufficiently bright to be studied. Yet the outskirts of galaxies - a galaxy's interface with the intergalactic medium and the location of critical processes such as pristine gas accretion and interactions with neighbouring galaxies - remain poorly explored. This volume brings together contributions on, among other topics, the stellar populations, star formation properties, metallicities and multi-phase gas in the outskirts of the Milky Way, local galaxies and beyond.

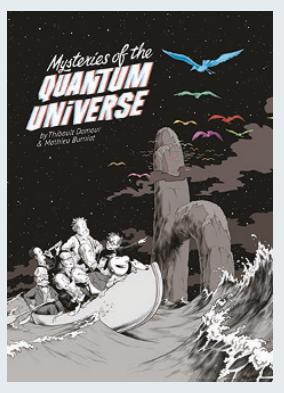

\section{Mysteries of the Quantum Universe}

By Thibault Damour and Mathieu Burniat PARTICULAR BOOKS: 2017. 160PP. E17.99

In the first panel of this graphic novel, Bob and his dog Rick (a more science-obsessed version of Tintin and Snowy) are being launched into space. A few panels and a meteor-induced tragedy later, Bob and his now stuffed (and technically dead) dog Rick are off on a new adventure to the land of the quanta. Together, they traverse the quantum universe, meet those that contributed to its physical description (including Einstein, Planck, Heisenberg and Schrödinger) and learn its secrets. In a parallel quantum state, Rick the dog may even be alive!

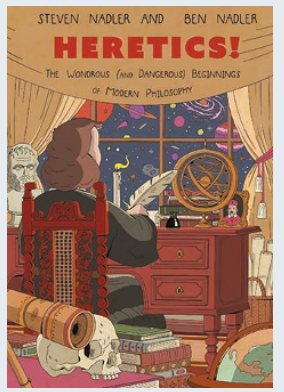

\section{Heretics!}

By Steven Nadler and Ben Nadler PRINCETON UNIVERSITY PRESS: 2017. 192PP. £18.95

Scientific progress from antiquity to today has been a procession of individuals willing to go against the tide to claim that thunder is only an electrical discharge or that light is made out of particles. Heretics! Taking a broader view of modern philosophy, including contributions from the likes of Galileo Galilei and Isaac Newton, this graphic novel describes the troubles (including but not limited to torture, prison and even death) of 17th century thinkers who challenged authority. This book offers an excellent, easily digestible introduction to the history of modern philosophy and science. 